\title{
Identification and Characterization of a Cryptic Genomic Deletion-Insertion in EYA1 Associated with Branchio-Otic Syndrome
}

\author{
Hao Zheng, ${ }^{1,2}$ Jun Xu, ${ }^{3,4,5}$ Yu Wang, ${ }^{1}$ Yun Lin $\mathbb{D}^{3,4,5}$ Qingqiang Hu, ${ }^{1}$ Xing Li, ${ }^{1}$ Jiusheng Chu, ${ }^{1}$ \\ Changling Sun $\unrhd^{6},{ }^{6}$ Yongchuan Chai $\mathbb{D}^{3,4,5}$ and Xiuhong Pang ${ }^{1}{ }^{1}$ \\ ${ }^{1}$ Department of Otolaryngology-Head and Neck Surgery, Taizhou People's Hospital, The Fifth Affiliated Hospital of Nantong \\ University \& Clinical Hospital of Dalian Medical University, Taizhou, Jiangsu, China \\ ${ }^{2}$ Department of Clinical Medicine, Medical School of Nantong University, Nantong, Jiangsu, China \\ ${ }^{3}$ Department of Otolaryngology-Head and Neck Surgery, The Ninth People's Hospital, Shanghai Jiaotong University School \\ of Medicine, Shanghai, China \\ ${ }^{4}$ Ear Institute, Shanghai Jiaotong University School of Medicine, Shanghai, China \\ ${ }^{5}$ Shanghai Key Laboratory of Translational Medicine on Ear and Nose Diseases, Shanghai, China \\ ${ }^{6}$ Department of Otolaryngology-Head and Neck Surgery, Affiliated Hospital of Jiangnan University, Wuxi, Jiangsu, China
}

Correspondence should be addressed to Changling Sun; winterc126@sina.com, Yongchuan Chai; cycperfect@163.com, and Xiuhong Pang; pxhzxy@163.com

Received 23 February 2021; Accepted 19 March 2021; Published 7 April 2021

Academic Editor: Geng lin Li

Copyright (c) 2021 Hao Zheng et al. This is an open access article distributed under the Creative Commons Attribution License, which permits unrestricted use, distribution, and reproduction in any medium, provided the original work is properly cited.

Branchio-oto-renal spectrum disorder (BORSD) is characterized by hearing loss accompanied by ear malformations, branchial cysts, and fistulae, with (branchio-oto-renal syndrome (BORS)) or without renal abnormalities (BOS (branchio-otic syndrome)). As the most common causative gene for BORSD, dominant mutations in EYA1 are responsible for approximately $40 \%$ of the cases. In a sporadic deaf patient diagnosed as BOS, we identified an apparent heterozygous genomic deletion spanning the first four coding exons and one $5^{\prime}$ noncoding exon of EYA1 by targeted next-generation sequencing of 406 known deafness genes. Real-time PCR at multiple regions of EYA1 confirmed the existence of this genomic deletion and extended its $5^{\prime}$ boundary beyond the $5^{\prime}$-UTR. Whole genome sequencing subsequently located the $5^{\prime}$ and $3^{\prime}$ breakpoints to 19268 bp upstream to the ATG initiation codon and $3180 \mathrm{bp}$ downstream to exon 5. PCR amplification across the breakpoints in both the patient and his parents showed that the genomic alteration occurred de novo. Sanger sequencing of this PCR product revealed that it is in fact a GRCh38/hg38:chr8:g.71318554_71374171delinsTGCC genomic deletion-insertion. Our results showed that the genomic variant is responsible for the hearing loss associated with BOS and provided an example for deciphering such cryptic genomic alterations following pipelines of comprehensive exome/genome sequencing and designed verification.

\section{Introduction}

Branchio-oto-renal spectrum disorder (BORSD) characterized by malformations of the outer, middle, and inner ear associated with conductive, sensorineural, or mixed hearing loss, branchial cysts and fistulas, and renal abnormalities comprises branchio-oto-renal (BOR) syndrome (BOR1: \#113650, BOR2: \#610896) and branchio-otic syndrome (BOS) (BOS1: \#602588, BOS3: \#608389) [1, 2], two pheno- types that differ only by the presence or absence of renal abnormalities. BORSD affects about 1 in 40000 children including $2 \%$ of profoundly deaf children $[3,4]$. Same to other dominant disorders, the offspring of $\mathrm{BOR} / \mathrm{BOS}$ individuals are at a $50 \%$ risk of inheriting the pathogenic variant. Once the pathogenic variant has been identified in an affected family member, prenatal testing for a pregnancy or preimplantation genetic diagnosis (PGD) becomes possible $[5,6]$. 
The known disease-causing genes for BOR/BOS are EYA1 (\#601653; located in 8q13.3), SIX1 (\#601205; located in 14q23.1), and SIX5 (\#600963; located in 19q13.32), in which EYA1 is the most frequent gene responsible for about $40 \%$ of affected patients [7]. EYA1, the human homolog of the Drosophila eyes absent gene, acts as a protein phosphatase and transcriptional coactivator [8, 9]. Eya1 homozygousdeficient mice lack ears and kidneys, and Eyal heterozygousdeficient mice present with phenotypes resembling BOR syndrome $[10,11]$. The majority of disease-associated missense mutations cluster in the conserved C-terminal 271residue Eya domain (ED) of EYA1 (321-592 residues) [12]. Otherwise, the N-terminal domain of EYA1 (1-320 residues) is poorly conserved and can attenuate the catalytic activity of Eya to achieve transactivation when bound to a DNAbinding protein $[9,13]$. To date, more than 190 mutations in EYA1 have been found to be associated with BOR/BOS, of which copy number variants (CNVs) account for about 17.120\% (http://www.hgmd.cf.ac.uk/ac/all.php, last updated in April 2019) [7, 14, 15].

CNVs, DNA segments including deletions, duplications, and complex rearrangements which exceed $1 \mathrm{~kb}$, are a major source of genome diversity in human populations $[16,17]$ and have been implicated in a variety of human diseases and cancers $[18,19]$. Large CNVs, especially the heterozygous ones, however, were hard to be detected by the conventional mutation screening methods such as polymerase chain reaction (PCR) amplification and Sanger sequencing. With the wide application of various genome sequencing technologies, an increasing number of rare CNVs have been found to play a vital role in genetic etiology of hearing loss $[20,21]$. But so far, there is no recognized pipeline for the detection of cryptic genomic alterations that are hard to detect by conventional methods.

In this study, we reported how multiple genomic sequencing methods including targeted NGS, real-time PCR, WGS, and Sanger sequencing were applied comprehensively to identify a heterozygous 55618 bp genomic deletioninsertion of EYA1 gene in a sporadic patient with BOS. This may provide an example for deciphering such cryptic genomic alterations following pipelines of comprehensive exome/genome sequencing and designed verification.

\section{Materials and Methods}

2.1. Editorial Policies and Ethical Considerations. All subjects in this study gave written, informed consent to participate in this study. Identifying information will not be included in the manuscript unless the information is essential for scientific purposes. This study was approved by the Ethics Committee of Taizhou People's Hospital, the Fifth Affiliated Hospital of Nantong University, and was compliant with the Declaration of Helsinki.

2.2. Subjects and Clinical Examinations. The proband II-1 and his parents (I-1 and I-2) were recruited by the Department of Otolaryngology-Head and Neck Surgery, Taizhou People's Hospital, Jiangsu Province (Figure 1(a)). Comprehensive clinical history was taken, and a detailed physical examination was performed in all subjects with special attentions to audiological, branchial, renal, olfactory, cardiac, ophthalmologic, skeletal, mental, intestinal, and dermatologic abnormalities. The hearing loss was confirmed by otoscopy, pure-tone audiometry (PTA), immittance, distortion product otoacoustic emission (DPOAE), and auditory brainstem response (ABR). The malformation of the middle and inner ear was confirmed by highresolution CT (HRCT) and magnetic resonance imaging (MRI). Renal abnormalities were excluded by ultrasound and renal function test. Phenotypes of BOS/BOR were evaluated by the diagnostic criteria described previously [7]. Major criteria are branchial anomalies, deafness, preauricular pits, and renal anomalies. Minor criteria are external ear anomalies, middle ear anomalies, inner ear anomalies, preauricular tags, and others, including facial asymmetry and palate abnormalities.

\subsection{Targeted NGS of 406 Deafness Genes. Genomic DNA was} extracted from the whole blood using the Blood DNA Kit (TIANGEN BIOTECH, Beijing, China). Sequencing of all 406 deafness-related genes was completed by targeted next-generation sequencing (NGS) using the MyGenotics gene enrichment system (Panel1-V4, MyGenotics, Boston, MA, USA) and the Illumina HiSeq 2000 sequencer (Illumina, San Diego, CA, USA) as previously described (Supplementary Table S1) [22]. The reads were aligned to HG19 using the BWA software, and the variants were called using the Genome Analysis Toolkit (GATK), both with the default parameters. The copy numbers of related genes were obtained through CapCNV analysis followed by CNVkit protocol (https://cnvkit.readthedocs .io/en/stable/pipeline.html). SNVs and indels were presented using Variant Call Format (VCF) version 4.1 and annotated using the ANNOVAR software. Data analysis and bioinformatics processing were performed as previously described [22].

Possible pathogenic effect of the missense mutations was evaluated by computational tools including CADD, Exome Variant Server, gnomAD, MutationTaster, PolyPhen-2, 1000 genomes, PhastCons, Phylop, PROVEAN (cut-off score $<-2.5$ ), and SIFT (cut-off score $<0.05$ ).

2.4. Real-Time PCR. To verify the existence and explore approximate breakpoint position of the suspected heterozygous genomic deletion related to exons 1-5 in EYA1 found by targeted NGS of 406 deafness genes (Figure 2(a)), primers were designed in its upstream, middle, and downstream regions, including $5^{\prime}$-end upstream region, noncoding $5^{\prime}$-UTR, exon 2 , exon 5 , and exon 6 (Figure 2(b)). The primers were designed by the PRIME3 software online (http://frodo.wi.mit.edu/cgi-bin/primer3/ primer3_www.cgi). Real-time PCR was performed in the proband, his unaffected parents, and a normal-hearing control on the 7300 Real-Time PCR System (Applied Biosystems) using SYBR ${ }^{\circledR}$ Premix Ex Taq ${ }^{\text {TM }}$ (Takara Bio Company). Each reaction was repeated three times and the average $\mathrm{Ct}$ was recorded [21]. 


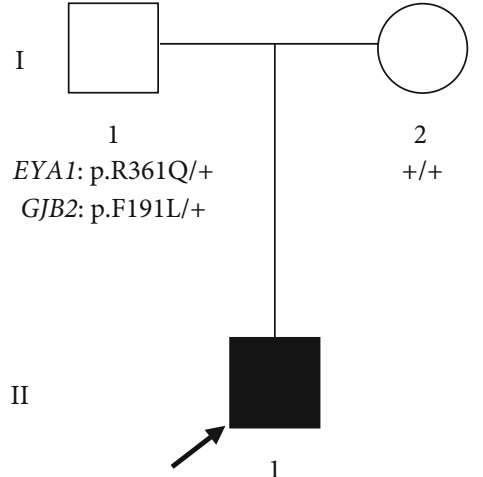

EYA1: genomic deletion-insertion/p.R361Q GJB2: p.F191L/+

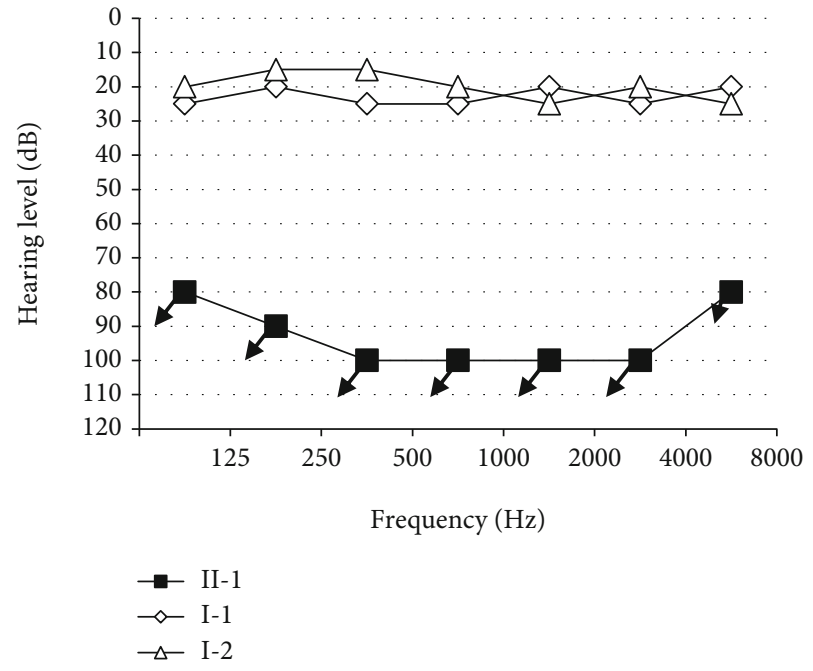

(a)
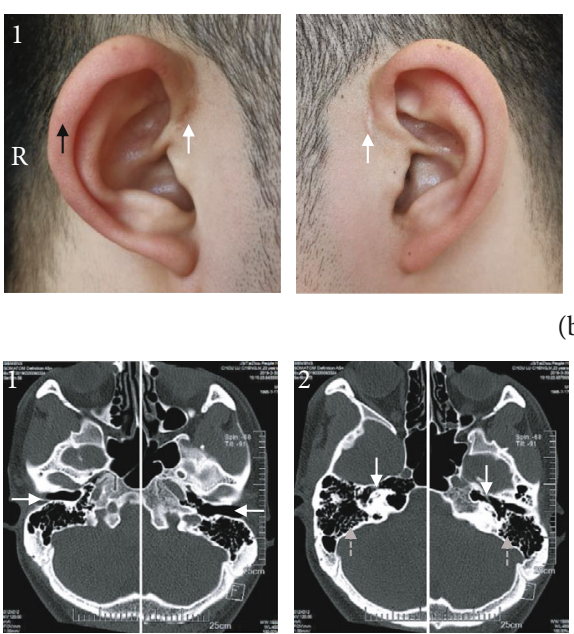

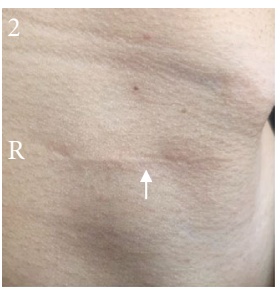

(b)
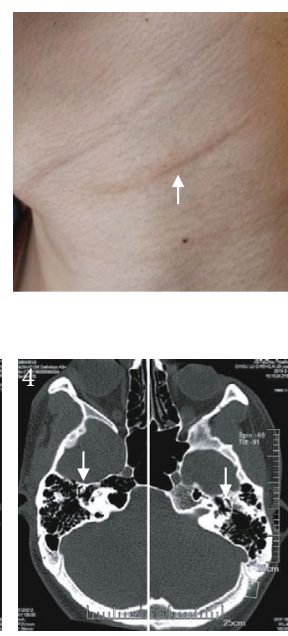

(c)

Figure 1: Pedigree, genotype, and phenotype characterization of the family. (a) Pedigree, genotype, and audiograms. Proband II-1 is pointed by the black arrow, and hearing loss is indicated by the black square. The audiograms showed profound sensorineural hearing loss in II-1 and normal hearing loss in his parents. (b) Right cup-shaped outer ear is showed by a black arrow in b1; two white arrows in b1 and b2 indicate bilateral surgical scars of preauricular fistula and cervical branchial cyst, respectively. (c) Findings in temporal HRCT: c1: bilateral lower external auditory canals; c2: white solid and grey dotted arrows indicate cochlear hypoplasia and overgasification of mastoid cells, respectively; c3: malformed semicircular canal; c4: deformed ossicular chain.

2.5. Whole Genome Sequencing. To judge whether the heterozygous deletion involved upstream genes or not and search for the exact breakpoint position of $5^{\prime}$ - and $3^{\prime}$-end, whole genome sequencing was selected (Figure 2(c)). Paired-end DNA libraries were prepared according to the manufacturer's instructions (Illumina TruSeq Library Construction). DNA libraries were sequenced on Illumina HiSeq $\mathrm{X}$ according to the manufacturer's instructions for paired-end $150 \mathrm{bp}$ reads. The average sequencing depth ranged from 31.35 to 57.77 , and $90.1 \%$ to $99.2 \%$ of whole genome were covered at least 20. Reads (without barcode) were aligned to HG19 using SpeedSeq. Single nucleotide variants, insertions, deletions, and indels calling were performed using Genome Analysis Toolkit v2.1. Structure variants and copy number variants were analyzed in SpeedSeq.
Annotations of single nucleotide variants, indels, structure variants, and copy number variants were performed with ANNOVAR [23].

2.6. Sanger Sequencing. To verify the results of WGS, a single PCR amplification was performed in the proband across the break junction (Figure 2(d)). The exact break junction and additional insertion were identified by sequencing of this PCR product. The same PCR amplification was used to detect the novel CNV in unaffected parents and a normalhearing control (forward primer F1: $5^{\prime}$-ATCTGTGGCCC CCAAATACTTC- ${ }^{\prime}$; reverse primer R1: $5^{\prime}$-AGGTCCT CTGCCCATTATTTGA-3'; PCR product size: $244 \mathrm{bp}$ ) (Figure $2(\mathrm{~d})$ ). In addition, a second PCR was performed 
(a) Targeted-NGS

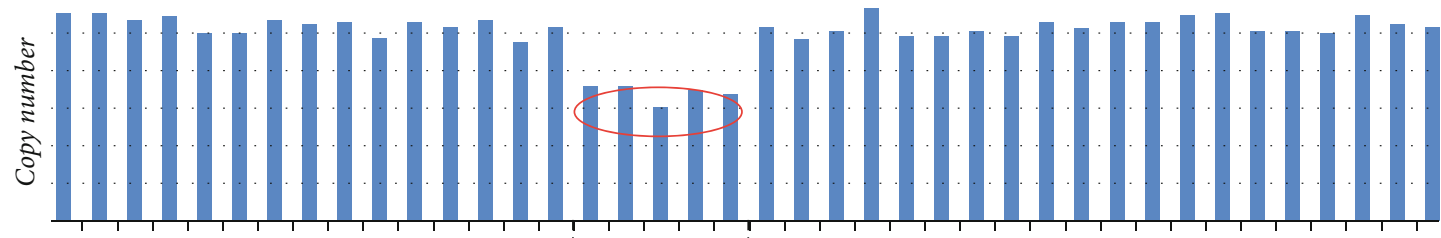

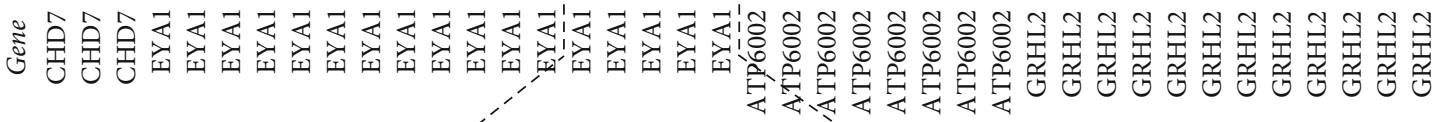

(b) Real-time PCR

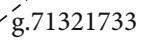

(c) WGS

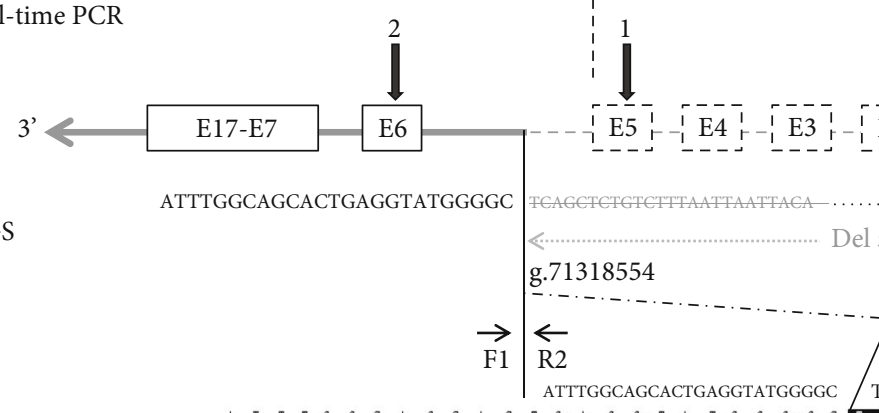
g.71356548

(d) Sanger sequencing

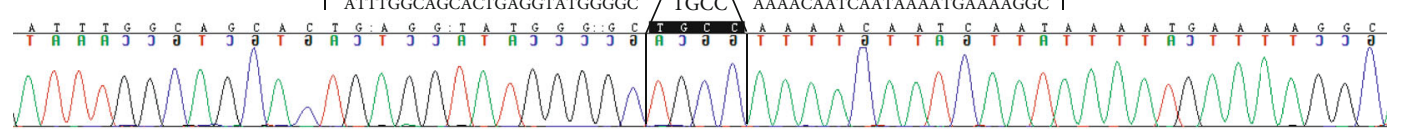

FIGURE 2: The process of identifying CNV. (a) The red oval indicates the region of deletion detected by targeted NGS. (b) Results of real-time PCR: 1 and 2 indicate copy number; black arrows: primer position; E: exon; left grey arrow: the $3^{\prime}$-end; right grey arrow: the $5^{\prime}$-end. (c) Grey words: region of deletion; F and R: forward and reverse primer; Ins: insertion. (d) Sequence diagram of $244 \mathrm{bp}$ PCR product. 4 bases between the two black vertical lines were additional insertion.

to amplify across the $5^{\prime}$ breakpoint from the wild-type allele (forward primer F2: $5^{\prime}$-TTAGACCAGACACAAA AGCAACTCC-3'; reverse primer R1: 5'-AGGTCCTCT GCCCATTATTTGA-3'; PCR product size: $364 \mathrm{bp}$ ) (Figure 2(d)), and a third PCR was performed to amplify across the $3^{\prime}$ breakpoint from the wild-type allele (forward primer F1: $5^{\prime}$-ATCTGTGGCCCCCAAATACTTC- $3^{\prime}$; reverse primer R2: 5'-AGAAAGGGATTTTCTAAAGCCATCA-3'; PCR product size: $559 \mathrm{bp}$ ) (Figure 2(d)). The CNV was determined as heterozygous if all PCR products were amplified and as homozygous if only the $244 \mathrm{bp}$ but not the $364 \mathrm{bp}$ and 559 bp products were amplified. No CNV was detected when only $364 \mathrm{bp}$ and $559 \mathrm{bp}$ were amplified. This CNV was subsequently screened in 400 ethnically matched normal controls (data not shown) and excluded benign CNV listing in the Database of Genomic Variants (http://dgv.tcag.ca/dgv/app/ about?ref=).

\section{Results}

3.1. Clinical Characteristics. The sporadic patient II-1 was found with bilateral congenital profound sensorineural hearing loss (Figure 1(a)), right cup-shaped outer ear, bilateral old surgical scars of congenital preauricular fistula, and cervical branchial cysts (Figure 1(b)). Bilateral lower external auditory canals, enlarged middle ear cavity, overgasification of mastoid cells, malformed ossicular chain, cochlear hypoplasia in immature apical turn and absence of the middle turn, malformed semicircular canals, and abnormal internal auditory canals were found by temporal bone HRCT (Figure 1(c)). Renal and other abnormalities were excluded after a series of detailed clinical examinations.

3.2. Screening for All Known Deafness Genes by Targeted NGS. Five heterozygous variants were submitted by targeted NGS of 406 known deafness genes in this patient: a genomic deletion spanning coding exon 2-6 in EYA1 (GRCh38/h38: chr8: 71321733-71356548) (Figure 2(a)), c.1082G $>$ A (p.Arg361Gln) in EYA1 (NM_000503.6), c.571T $>$ C (p.Phe191Leu) in GJB2 (NM_004004.6), c.2575C >G (p.Gln859Glu) in TCOF1 (NM_001135243), and c.685T>C (p.Tyr229His) in KARS (NM_001130089) (Figure 3). Possible pathogenic effect of the missense mutations was evaluated by computational tools (Table 1). Variants p.Arg361Gln (rs145219836) in EYA1 and p.Phe191Leu (rs397516878) in GJB2 were proved to be inherited from his unaffected father, while p.Gln859Glu (rs201043592) in TCOF1 and p.Tyr229His (rs150529876) in KARS were from his unaffected mother (Figure 3 and Table 1).

3.3. Verify the Existence of the Deletion by Real-Time PCR. To verify if the genomic deletion found by targeted NGS existed or not, the DNA segments in $5^{\prime}$-upstream region, $5^{\prime}$-UTR, exon 2 , exon 5 , and exon 6 were detected quantitatively, and their copy numbers were $1,1,1,1$, and 2 , respectively (Figure 2(b)). The genomic deletion was proved to exist and its $3^{\prime}$-end breakpoint located within exon 5-exon 6 . But where the $5^{\prime}$-end breakpoint was and whether the 


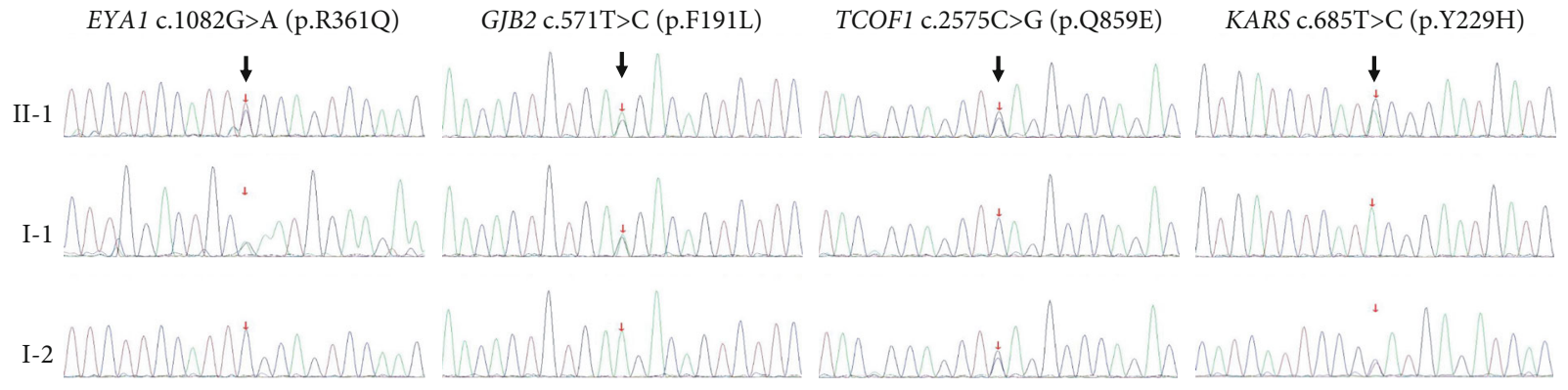

Figure 3: Sequencing diagram of 4 missense variants in patient II-1, unaffected parents I-1/I-2. Black arrow: changed base position.

deletion involved upstream genes or not need further exploration.

\subsection{Breakpoints of the Deletion Identified by WGS. To explore} where the $5^{\prime}$-end breakpoint was and whether the deletion involved upstream genes or not, WGS was selected. 55618 bp genomic deletion located in chromosome 8q13.3 was identified from g.71318554 to g.71374171 (19268 bp upstream to initiation codon ATG and $3180 \mathrm{bp}$ downstream to exon 5), which involved $5^{\prime}$ upstream, noncoding $5^{\prime}$-UTR and exon 1 , coding exons $2-5$, intron 1-4, and partial intron 5 (Figure 2(c)).

3.5. True Genomic CNV Was Identified by Sanger Sequencing. The breakpoints detected by WGS were verified exactly the same by amplification of the 244 bp product across $5^{\prime}$ and $3^{\prime}$ breakpoints. Notably, an additional $4 \mathrm{bp}$ insertion not detected by WGS was identified by Sanger sequencing. So a novel deletion-insertion variant GRCh38/hg38:chr8:g.71318554_ 71374171delinsTGCC spanned $5^{\prime}$-UTR, exons 1 to 5, was identified in our study (Figure 2(d)). $559 \mathrm{bp}$ and $364 \mathrm{bp}$ products amplified successfully suggested that the deletioninsertion variant was a heterozygous one. Meanwhile, these three fragments (559 bp, $364 \mathrm{bp}$, and $244 \mathrm{bp}$ ) also amplified in unaffected parents and one normal-hearing control, only fragments of $559 \mathrm{bp}$ and $364 \mathrm{bp}$ but $244 \mathrm{bp}$ amplified successfully in them (Figure 4).

Moreover, the novel deletion-insertion variant in EYA1 was not found in 400 ethnically matched normal controls and was ruled out as a benign CNV listed in the Database of Genomic Variants (DGV, http://dgv.tcag.ca/dgv/app/ about?ref=).

\section{Discussion}

Hearing loss is one of the major disabilities worldwide, which is often induced by loss of sensory hair cells in the inner ear cochlea [24-28]. Hearing loss could be caused by genetic factors, aging, chronic cochlear infections, infectious diseases, ototoxic drugs, and noise exposure [29-37]; and genetic factors account for more than $60 \%$ of hearing loss. According to phenotypes of BOS/BOR evaluated by the diagnostic criteria described previously [7], BOS was diagnosed in our sporadic patient with three major criteria of branchial anomaly, deafness, and preauricular pits and three minor criteria of external, middle, and inner ear anomalies. In contrast to the deformities of the ear reported in a previous study which showed atresia or stenosis in the external auditory canal, reduction in size of the middle ear space and hypoplastic mastoid cells [2], enlarged middle ear cavity, and overgasification of mastoid cells were discovered in our study (Figure 1(c)). In addition, the lower position of bilateral external auditory canals was first reported. High heterogeneity of phenotypes in BOR spectrum disease was further confirmed by our findings [7].

A heterozygous missense variant p.Arg361Gln and a genomic CNV GRCh38/hg38:chr8:g.71318554_71374171delins TGCC in EYA1 were identified simultaneously in our study. The former was suggested to be a nonpathogenic variant due to inheriting from his phenotypically normal father and benign predicted result of PolyPhen-2, PROVEAN, and SIFT (Figures 1 and 3, Table 1). The latter, a novel genomic deletion-insertion variant spanned $5^{\prime}$-UTR, exons 1 to 5 , involving $5^{\prime}$-UTR and N-terminal domain, was very likely to be a pathogenic mutation, due to it being not found in his phenotypically normal parents (Figure 4) and 400 ethnically matched normal controls, and was ruled out as a benign CNV listed in the DGV. De novo mutation was proved by the parental origin of variants p.Arg361Gln in EYA1, p.Phe191Leu in GJB2, p.Gln859Glu (rs201043592) in TCOF1, and p.Tyr229His in KARS (Figures 1 and 3, Table 1). This result further proved previous opinion about high de novo rate in EYA1 [38].

To date, two pathogenic mutations c.241C $>\mathrm{T}$ (p.Gln81Ter) and c.229C>T (p.Arg77Ter) (NM_000503.6) involving exons 1-5 are reported in previous study. They are predicted to be pathogenic due to loss of function from truncated or absent protein (https://www.ncbi.nlm.nih.gov/ clinvar/variation). These two pathogenic mutations were included in the range of the novel genomic CNV discovered in our study. Haploinsufficiency seems to be the most likely explanation for BOR-related phenotypes in cases with nonsense and large deletions leading to similar disease phenotypes $[39,40]$. In addition, the presence of the $\mathrm{N}$-terminal domain significantly attenuates the phosphatase activity of Eya [9]. It has been proposed that the ED domain of Eya acts as an autoinhibitor of the transactivation potential of the $\mathrm{N}$ terminal domain [41]. A decreased ability in binding to special proteins of $\mathrm{N}$-terminal domain caused by haploinsufficiency led to a decline in transactivation of EYA1, which was very likely the pathogenic mechanism of the genomic deletion-insertion mutation identified in our study $[9,13]$. 


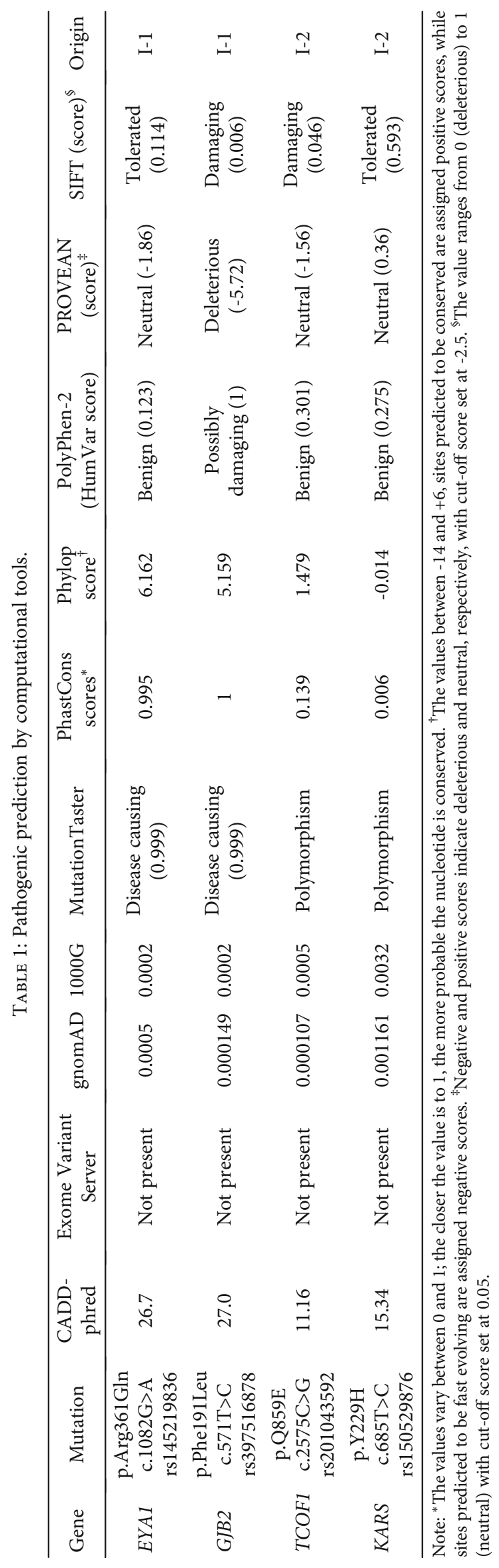




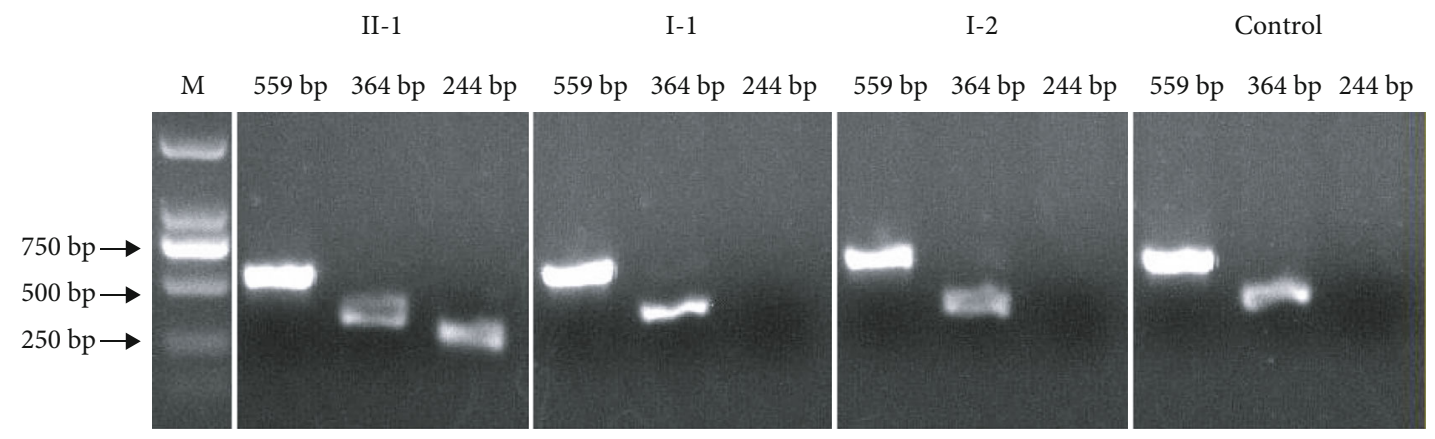

FIGURE 4: Electrophoresis gel figure of $244 \mathrm{bp}, 364 \mathrm{bp}$, and $559 \mathrm{bp}$ PCR product in patient II-1, unaffected parents I-1/I-2, and control. M: marker.

So far, no specialized detection scheme was considered reasonable for rapid and economical detection of cryptic genomic CNVs. In our study, by applying multiple genomic testing methods step by step to identify a potential heterozygous genomic CNV (Figure 2), a rational pipeline for detecting cryptic genomic CNVs appeared to have been successfully established. Since most of the pathogenic mutations are located in the coding region and its flanking sequences and the cost of targeted NGS and WES is relatively lower than that of WGS, researchers often choose one of them to search for pathogenic mutations first. A potential heterozygous genomic deletion involving exon 1-exon 5 of EYA1 was reported by targeted NGS (Figure $2(\mathrm{a})$ ). To verify whether the deletion existed and the range of $5^{\prime}$ - and $3^{\prime}$-end breakpoints, real-time PCR was considered as the preferred method for quantification [21]. Unfortunately, while confirming the presence of the deletion, $5^{\prime}$-end breakpoint region could not be evaluated by real-time PCR (Figure 2(b)). Considering economy, accuracy, time, and manpower saving, WGS was selected to detect the exact breakpoints. Encouragingly, the position of $5^{\prime}$ - and $3^{\prime}$-end breakpoints detected by WGS was proved perfectly accurate by Sanger sequencing (Figures 2(c) and 2(d)). However, if there was no verification by Sanger sequencing, the "TGCC" insertion that WGS failed to detect would have been missed (Figure 2(d)). In the process of detection, we discovered that all molecular detection methods applied above had their own special advantages and disadvantages; reasonable and comprehensive application of them was of great significance for the efficient detection of cryptic heterozygous genomic CNV. Targeted NGS and WES only detected exons and their flanking sequences, but not noncoding regions, so they can only provide clues for the possible existence of CNVs. Fortunately, due to no restriction on the region of primers for quantitative detection, real-time PCR can well make up for the defects of targeted NGS and WES and can confirm the existence of CNV and the range of its breakpoints no matter in coding or noncoding regions. When the region of $\mathrm{CNV}$ breakpoints cannot be determined, WGS can be selected to find the breakpoints accurately in the genomic level. However, given that the read length of WGS is about 150-200 bp, short fragment may be missed for complex CNVs, such as deletion- insertion. As the most accurate sequencing technology, Sanger sequencing can detect each base missed by WGS in targeted amplified region and thus dig out the true genomic CNVs. So we suggested that the reasonable application of the above sequencing methods step by step can be used as a pipeline for detection of cryptic genomic CNVs in our future work.

According to the parental origin and pathogenic prediction of computational tools, missense p.Arg361Gln in EYA1, p.Gln859Glu (rs201043592) in TCOF1, and p.Tyr229His in KARS were considered as benign variants (Figures 1 and 3, Table 1). Although p.Phe191Leu in GJB2 was listed as uncertain significance in ClinVar (https://www.ncbi.nlm .nih.gov/clinvar), it was regarded as a recessive inherited pathogenic one in our daily counseling work according to functional study [42]. Unfortunately, biallelic pathogenic mutations c.235delC and c.176_191del of GJB2 were identified in his girlfriend with nonsyndromic profound hearing loss. According to the law of autosomal recessive inheritance, the offspring of the proband and his girlfriend is at a $50 \%$ risk of inheriting biallelic pathogenic variants of GJB2. To EYA1, also $50 \%$ of their offspring should be affected with BOR spectrum diseases in an autosomal dominant way. Therefore, it was of great significance to identify the genetic pathogenic factors for them, which provides a theoretical basis for prenatal diagnosis or PGD to get healthy offspring. Given that the chance of conceiving healthy offspring naturally was only $25 \%$, we suggested that PGD technology was their best choice.

\section{Conclusion}

In conclusion, a novel heterozygous de novo genomic deletion-insertion in EYA1, GRCh38/hg38:chr8:.71318554_ 71374171delinsTGCC, was very likely the pathogenic cause for the patient with BOS due to a decline in transactivation of EYA1 resulting from haploinsufficiency. Through genetic counseling, the disease from EYA1 and GJB2 in the offspring of the patient can be avoided in the process of subsequent reproduction. Our results provided an example for deciphering such cryptic genomic alterations following pipelines of comprehensive exome/genome sequencing and designed verification. 


\section{Data Availability}

All the sequencing data supporting the conclusions of the study can be obtained by contacting Xiuhong Pang via email (pxhzxy@163.com).

\section{Conflicts of Interest}

The authors declare no conflict of interest.

\section{Authors' Contributions}

$\mathrm{XP}, \mathrm{YC}$, and CS conceived and designed the experiments. $\mathrm{HZ}$, JX, YL, and YW performed the experiments. XP, YC, $\mathrm{HZ}$, and CS analyzed the data. XP, QH, XL, and JC collected the samples and reviewed the phenotypes. $\mathrm{HZ}$ and XP wrote the paper. All authors reviewed the manuscript. Hao Zheng, Jun $\mathrm{Xu}$, and $\mathrm{Yu}$ Wang contributed equally to this work and share first authorship.

\section{Acknowledgments}

We would like to thank the family for agreeing to include their details. The Government of China is also acknowledged for providing research grant. This research was supported by grants from the National Natural Science Foundation of China (81700920 to X.P.), the Natural Science Foundation of Jiangsu Province (BK20191229 to X.P.), the Fifth "333 Project" Scientific Research Foundation of Jiangsu Province (BRA2019192 to X.P.), the Medical Scientific Research Project from Health and Family Planning Commission of Jiangsu Province (H201666 to X.P.), the Science and Technology Commission of Shanghai Municipality (14DZ2260300 to Y.C.), and the Medical Top-Notch Young Talents Program of Wuxi (BJ2020043 to C.S.).

\section{Supplementary Materials}

Supplementary Table S1: 406 causative genes for deafness were screened by targeted NGS. (Supplementary Materials)

\section{References}

[1] A. Kochhar, S. M. Fischer, W. J. Kimberling, and R. J. Smith, "Branchio-oto-renal syndrome," American Journal of Medical Genetics. Part A, vol. 143A, no. 14, pp. 1671-1678, 2007.

[2] R. J. H. Smith, Branchiootorenal spectrum disorder, M. P. Adam, H. H. Ardinger, R. A. Pagon, S. E. Wallace, and B. LJH, Eds., GeneReviews((R)), Seattle (WA), 1993.

[3] F. C. Fraser, J. R. Sproule, F. Halal, and J. M. Optiz, "Frequency of the branchio-oto-renal (BOR) syndrome in children with profound hearing loss," American Journal of Medical Genetics, vol. 7, no. 3, pp. 341-349, 1980.

[4] C. Stinckens, L. Standaert, J. W. Casselman et al., "The presence of a widened vestibular aqueduct and progressive sensorineural hearing loss in the branchio-oto-renal syndrome. A family study," International Journal of Pediatric Otorhinolaryngology, vol. 59, no. 3, pp. 163-172, 2001.

[5] S. Kumar, K. Deffenbacher, C. W. R. J. Cremers, G. van Camp, and W. J. Kimberling, "Branchio-oto-renal syndrome: identification of novel mutations, molecular characterization, muta- tion distribution, and prospects for genetic testing," Genetic Testing, vol. 1, no. 4, pp. 243-251, 1997.

[6] T. Matsunaga, M. Okada, S. Usami, and T. Okuyama, "Phenotypic consequences in a Japanese family having branchiooto-renal syndrome with a novel frameshift mutation in the gene EYA1," Acta Otolaryngol, vol. 127, no. 1, pp. 98-104, 2007.

[7] E. H. Chang, M. Menezes, N. C. Meyer et al., "Branchio-otorenal syndrome: the mutation spectrum in EYA1 and its phenotypic consequences," Human Mutation, vol. 23, no. 6, pp. 582-589, 2004.

[8] P. X. Xu, J. Cheng, J. A. Epstein, and R. L. Maas, “Mouse Eya genes are expressed during limb tendon development and encode a transcriptional activation function," Proceedings of the National Academy of Sciences of the United States of America, vol. 94, no. 22, pp. 11974-11979, 1997.

[9] J. P. Rayapureddi and R. S. Hegde, "Branchio-oto-renal syndrome associated mutations in eyes absent 1 result in loss of phosphatase activity," FEBS Letters, vol. 580, no. 16, pp. 3853-3859, 2006.

[10] P. X. Xu, J. Adams, H. Peters, M. C. Brown, S. Heaney, and R. Maas, "Eya1-deficient mice lack ears and kidneys and show abnormal apoptosis of organ primordia," Nature Genetics, vol. 23, no. 1, pp. 113-117, 1999.

[11] P. X. Xu, W. Zheng, C. Laclef et al., "Eya1 is required for the morphogenesis of mammalian thymus, parathyroid and thyroid," Development, vol. 129, no. 13, pp. 3033-3044, 2002.

[12] S. Abdelhak, V. Kalatzis, R. Heilig et al., "Clustering of mutations responsible for branchio-oto-renal (BOR) syndrome in the eyes absent homologous region (eyaHR) of EYA1," Human Molecular Genetics, vol. 6, no. 13, pp. 2247-2255, 1997.

[13] K. Ikeda, Y. Watanabe, H. Ohto, and K. Kawakami, "Molecular interaction and synergistic activation of a promoter by Six, Eya, and Dach proteins mediated through CREB binding protein," Molecular and Cellular Biology, vol. 22, no. 19, pp. 67596766, 2002.

[14] E. Razmara, F. Bitarafan, E. Esmaeilzadeh-Gharehdaghi, N. Almadani, and M. Garshasbi, "The first case of NSHL by direct impression on EYA1 gene and identification of one novel mutation in MYO7A in the Iranian families," Iranian Journal of Basic Medical Sciences, vol. 21, no. 3, pp. 333-341, 2018.

[15] Y. H. Ahn, C. Lee, N. K. D. Kim et al., "Targeted exome sequencing provided comprehensive genetic diagnosis of congenital anomalies of the kidney and urinary tract," Journal of Clinical Medicine, vol. 9, no. 3, p. 751, 2020.

[16] L. Feuk, A. R. Carson, and S. W. Scherer, "Structural variation in the human genome," Nature Reviews. Genetics, vol. 7, no. 2, pp. 85-97, 2006.

[17] The 1000 Genomes Project Consortium, P. H. Sudmant, T. Rausch et al., "An integrated map of structural variation in 2,504 human genomes," Nature, vol. 526, no. 7571, pp. $75-81,2015$.

[18] C. T. Watson, M. B. Tomas, A. J. Sharp, and H. C. Mefford, "The genetics of microdeletion and microduplication syndromes: an update," Annual Review of Genomics and Human Genetics, vol. 15, no. 1, pp. 215-244, 2014.

[19] S. Girirajan, C. D. Campbell, and E. E. Eichler, "Human copy number variation and complex genetic disease," Annual Review of Genetics, vol. 45, no. 1, pp. 203-226, 2011. 
[20] X. Pang, Y. Chai, L. He et al., “A 7666-bp genomic deletion is frequent in Chinese Han deaf patients with non-syndromic enlarged vestibular aqueduct but without bi-allelic SLC26A4 mutations," International Journal of Pediatric Otorhinolaryngology, vol. 79, no. 12, pp. 2248-2252, 2015.

[21] X. Pang, H. Luo, Y. Chai et al., "A 1.6-Mb microdeletion in chromosome 17q22 leads to NOG-related symphalangism spectrum disorder without intellectual disability," PLoS One, vol. 10, no. 3, article e0120816, 2015.

[22] L. He, X. Pang, H. Liu, Y. Chai, H. Wu, and T. Yang, "Targeted next-generation sequencing and parental genotyping in sporadic Chinese Han deaf patients," Clinical Genetics, vol. 93, no. 4, pp. 899-904, 2018.

[23] Z. Cen, Z. Jiang, Y. Chen et al., "Intronic pentanucleotide TTTCA repeat insertion in the SAMD12 gene causes familial cortical myoclonic tremor with epilepsy type 1," Brain, vol. 141, no. 8, pp. 2280-2288, 2018.

[24] Y. Liu, J. Qi, X. Chen et al., "Critical role of spectrin in hearing development and deafness," Science Advances, vol. 5, no. 4, article eaav7803, 2019.

[25] Y. Wang, J. Li, X. Yao et al., "Loss of CIB2 causes profound hearing loss and abolishes mechanoelectrical transduction in mice," Frontiers in Molecular Neuroscience, vol. 10, p. 401, 2017.

[26] J. Qi, Y. Liu, C. Chu et al., “A cytoskeleton structure revealed by super-resolution fluorescence imaging in inner ear hair cells," Cell Discovery, vol. 5, no. 1, p. 12, 2019.

[27] Z. H. He, S. Y. Zou, M. Li et al., "The nuclear transcription factor FoxG1 affects the sensitivity of mimetic aging hair cells to inflammation by regulating autophagy pathways," Redox Biology, vol. 28, p. 101364, 2020.

[28] J. Qi, L. Zhang, F. Tan et al., "Espin distribution as revealed by super-resolution microscopy of stereocilia," American Journal of Translational Research, vol. 12, no. 1, pp. 130-141, 2020.

[29] C. Zhu, C. Cheng, Y. Wang et al., "Loss of ARHGEF6 causes hair cell stereocilia deficits and hearing loss in mice," Frontiers in Molecular Neuroscience, vol. 11, p. 362, 2018.

[30] S. Gao, C. Cheng, M. Wang et al., "Blebbistatin inhibits neomycin-induced apoptosis in hair cell-like HEI-OC-1 cells and in cochlear hair cells," Frontiers in Cellular Neuroscience, vol. 13, 2020.

[31] Y. Zhang, W. Li, Z. He et al., "Pre-treatment with fasudil prevents neomycin-induced hair cell damage by reducing the accumulation of reactive oxygen species," Frontiers in Molecular Neuroscience, vol. 12, p. 264, 2019.

[32] F. Qian, X. Wang, Z. Yin et al., "The slc $4 a 2 b$ gene is required for hair cell development in zebrafish," Aging (Albany NY), vol. 12, no. 19, pp. 18804-18821, 2020.

[33] H. Zhou, X. Qian, N. Xu et al., "Disruption of Atg7-dependent autophagy causes electromotility disturbances, outer hair cell loss, and deafness in mice," Cell Death \& Disease, vol. 11, no. 10, p. 913, 2020.

[34] C. Cheng, Y. Wang, L. Guo et al., "Age-related transcriptome changes in Sox2+ supporting cells in the mouse cochlea," Stem Cell Research \& Therapy, vol. 10, no. 1, p. 365, 2019.

[35] Z. He, L. Guo, Y. Shu et al., "Autophagy protects auditory hair cells against neomycin-induced damage," Autophagy, vol. 13, no. 11, pp. 1884-1904, 2017.

[36] W. Liu, X. Xu, Z. Fan et al., "Wnt signaling activates TP53induced glycolysis and apoptosis regulator and protects against cisplatin-induced spiral ganglion neuron damage in the mouse cochlea," Antioxidants \& Redox Signaling, vol. 30, no. 11, pp. 1389-1410, 2019.

[37] Y. Zhang, S. Zhang, Z. Zhang et al., "Knockdown of Foxg1 in Sox9+ supporting cells increases the trans-differentiation of supporting cells into hair cells in the neonatal mouse utricle," Aging (Albany NY), vol. 12, no. 20, pp. 19834-19851, 2020.

[38] T. L. Stockley, R. Mendoza-Londono, E. J. Propst, S. Sodhi, L. Dupuis, and B. C. Papsin, "A recurrent EYA1 mutation causing alternative RNA splicing in branchio-oto-renal syndrome: implications for molecular diagnostics and disease mechanism," American Journal of Medical Genetics. Part A, vol. 149A, no. 3, pp. 322-327, 2009.

[39] N. Huang, I. Lee, E. M. Marcotte, and M. E. Hurles, "Characterising and predicting haploinsufficiency in the human genome," PLoS Genetics, vol. 6, no. 10, article e1001154, 2010.

[40] P. D. Brophy, F. Alasti, B. W. Darbro et al., "Genome-wide copy number variation analysis of a branchio-oto-renal syndrome cohort identifies a recombination hotspot and implicates new candidate genes," Human Genetics, vol. 132, no. 12, pp. 1339-1350, 2013.

[41] S. J. Silver, E. L. Davies, L. Doyon, and I. Rebay, "Functional dissection of eyes absent reveals new modes of regulation within the retinal determination gene network," Molecular and Cellular Biology, vol. 23, no. 17, pp. 5989-5999, 2003.

[42] C. Ambrosi, A. E. Walker, A. D. DePriest et al., "Analysis of trafficking, stability and function of human connexin 26 gap junction channels with deafness-causing mutations in the fourth transmembrane helix," PLoS One, vol. 8, no. 8, article e70916, 2013. 